\title{
Modèle dynamique d'électrobroche en rotation et prédiction de la stabilité de la coupe
}

\author{
Vincent Gagnol ${ }^{1, a}$, Belhassen Chedli Bouzgarrou ${ }^{1}$, Pascal Ray ${ }^{1}$ et Christian \\ BARRA $^{2}$ \\ 1 LAMI - Laboratoire de Mécanique et Ingénierie, BP 265, 63175 Aubière Cedex, France \\ 2 PCI-SCEMM, rue Copernic, 42000 Saint-Étienne, France
}

Reçu le 15 mars 2007, accepté le 6 juillet 2007

\begin{abstract}
Résumé - La prédiction des conditions de stabilité en usinage est d'une grande importance pour les opérations de fraisage à grande vitesse. Dans cet article un modèle dynamique de broche UGV est élaboré sur la base de la théorie de la dynamique des rotors. Les analyses dynamiques du modèle montrent une dépendance forte du comportement dynamique de la broche par rapport à sa vitesse de rotation. Une prédiction précise des conditions de stabilité en usinage doit nécessairement prendre en compte ces propriétés du comportement. En introduisant la fonction de transfert en bout d'outil de la broche, obtenue par le modèle, dans l'approche analytique d'analyse de la stabilité de la coupe d'Altintas et Budak, un nouveau diagramme de lobes de stabilité est élaboré. Chaque point de ce diagramme de lobe de stabilité est calculé sur la base des propriétés effectives de la broche à la fréquence de rotation considérée. Les limites de stabilité prédites par notre approche indiquent des variations significatives de stabilité, et permettent une prédiction plus précise des conditions d'usinage sans broutement. Des essais de coupe sont réalisés et permettent de valider expérimentalement les résultats en terme de stabilité obtenus par le modèle.
\end{abstract}

Mots clés : Comportement dynamique / broche ugv / stabilité de la coupe / lobes de stabilité

\begin{abstract}
Spindle rotor-dynamics model and high-speed milling stability prediction. Prediction of stable cutting conditions is a critical requirement for high-speed milling operations. In this paper, a high-speed spindle-bearing system dynamic model is elaborated on the basis of rotor dynamics prediction. It is shown that dynamic behaviour dependencies on the rotation speed range have to be taken into account for accurate stability prediction. By integrating the proposed speed dependent spindle transfer function in the chatter vibration stability approach of Altintas and Budak, a dynamic stability lobes diagram is predicted. The proposed method allows establishing a new stability lobes diagram that takes into account the dependency of dynamic behaviour on spindle speed. Significant changes are observed on the stability limits, constructed using the model, and allow accurate prediction of cutting conditions to be done. Finally, experiments are performed in order to validate the chatter boundaries prediction in practice.
\end{abstract}

Key words: Spindle dynamics / hsm spindle / chatter / stability lobes

\section{Introduction}

L'Usinage Grande Vitesse (UGV) est une technologie utilisée pour augmenter la productivité et réduire les coûts de production. La qualité de la surface usinée est souvent associée au diagramme de lobe de stabilité, qui définit les conditions de coupe conduisant à un usinage stable ou instable. Pendant un usinage, le phénomène

\footnotetext{
${ }^{a}$ Auteur pour correspondance:

Vincent.Gagnol@ifma.fr
}

de broutement peut se produire pour des conditions spécifiques d'épaisseur de copeau et de vitesse de broche. L'étude de ce phénomène représente un enjeu important puisqu'il peut conduire à l'endommagement de l'outil, de la broche ou de la pièce. De nombreuses études ont été réalisées depuis les années 50 sur le phénomène de broutement régénératif comme ceux de Tobias et Fishwick [1], Tlusty et Polacek [2], Merrit [3] et Altintas [4].

Les hypothèses sous-jacentes à ces modèles reposent sur l'indépendance du comportement dynamique d'une 

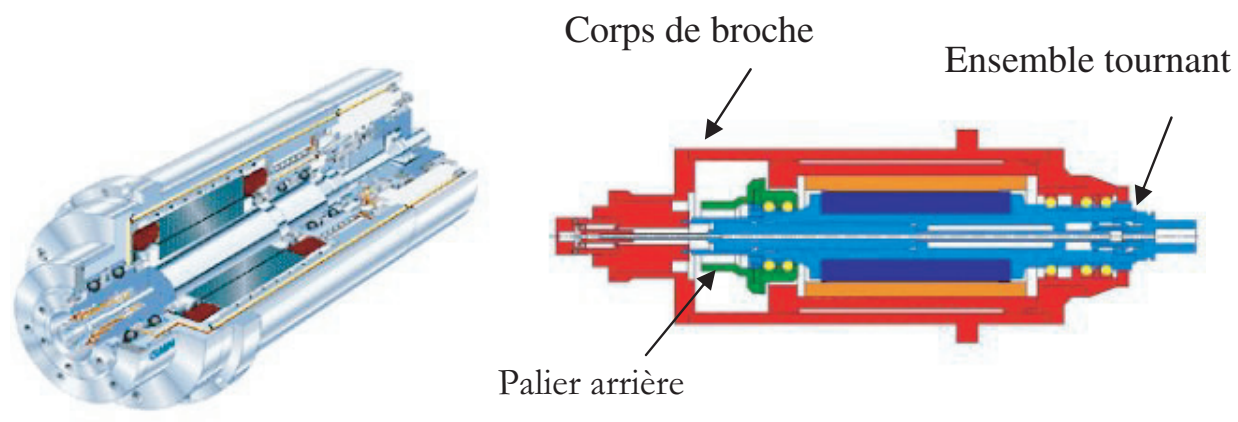

Fig. 1. Broche à roulement UGV.

broche vis-à-vis de sa fréquence de rotation sur l'ensemble de la gamme de vitesse considérée. La dépendance du comportement dynamique de broche et de la coupe n'est pas modélisée.

Les propriétés dynamiques d'une broche sont influencées par un grand nombre de facteurs comme les caractéristiques du porte-outil (Agapiou et al. [5]), la géométrie de l'arbre de broche et du système de serrage, Smith et al. [6], ainsi que par les rigidités et amortissements apportés par les roulements. La plupart de ces facteurs sont indépendants de la vitesse de rotation de la broche contrairement à la rigidité des roulements qui change avec la précharge et la vitesse de broche. W. Wang et C. Chang [7] ont réalisé une modélisation élémentsfinis (FEM) d'une broche sur deux roulements par des poutres de Rayleigh sans prendre en compte les effets liés aux grandes vitesses de rotation. Leurs résultats montrent l'importance de la variation d'angle de contact interne au roulement sur les modes de vibrations élevés. M.A. Alfares et A. Elsharkawy [8] ont présenté les effets de la précharge axiale des roulements à contact oblique sur les propriétés dynamiques d'une broche. Dans notre article, les rigidités des roulements sont modélisées par des matrices $6 \times 6$ selon la formulation développée par T.C. Lim [9] et B.R. Jorgensen [10]. Les modèles sont ensuite intégrés au modèle élément-fini de la broche.

La prédiction précise que les propriétés dynamiques d'une broche nécessitent d'évaluer les caractéristiques de dépendance du comportement de la broche vis-à-vis de sa fréquence de rotation. De nombreux travaux ont montré l'interaction qui existait entre la fréquence de rotation et les aspects thermiques dans une broche [10-13]. J.L. Stein et J.F. Tu [11], B. Bossmanns et J.F. Tu [12] ont représenté ce phénomène par une boucle fermée reliant la précharge à la dilatation des roulements. Un algorithme d'estimation de la précharge en conditions d'usinage a été développé par S.A. Spiewak et T. Nickel [13] à partir de mesures expérimentales de vibration. Ils montrent que les modifications comportementales consécutives à des dilatations thermiques peuvent être modélisées par l'évaluation précise des variations géométriques au sein des roulements. Il est possible de s'affranchir de ces considérations sur les électrobroches UGV récentes qui disposent de dispositifs hydrauliques ou à ressorts maintenant la précharge axiale des roulements constante malgré les dilatations thermiques.
Faassen [14] et Schmitz [15] proposent de considérer les propriétés de dépendance du comportement dynamique de broche en fonction de sa vitesse de rotation sur la base de l'identification de fonctions de réponse en fréquence de la broche à différentes vitesses de rotation. Cependant leur méthode est difficilement industrialisable en raison des temps trop longs d'immobilisation de la machine nécessaires à cette caractérisation. De plus, une caractérisation modale expérimentale par choc au marteau est mal adaptée pour fournir une information fiable des propriétés dynamiques d'un arbre tournant.

Dans cet article une approche originale d'analyse de la stabilité de la coupe en fraisage est présentée. Un modèle semi-analytique de broche basé sur la dynamique des rotors combiné à la méthode des éléments-finis est élaboré. La deuxième partie décrit les données géométriques et technologiques des broches nécessaires à l'élaboration du modèle. Le modèle EF est construit sur l'assemblage du modèle de l'ensemble tournant et des modèles des roulements. Un élément spécial de poutre rotor a été développé [16] et est implémenté. Les matrices de rigidité des roulements sont calculées, sur la base des travaux de T.C. Lim [9], autour d'un point de fonctionnement statique caractérisé par la précharge initiale.

Dans la section 3, l'analyse du comportement de la broche est présentée sur la base de simulations numériques du modèle. On montre, en particulier, que les propriétés dynamiques d'une broche en rotation sont dépendantes de la fréquence de rotation. Le modèle élaboré se démarque ainsi des modèles courants de broche construits sur la base des propriétés dynamiques de broche non tournante.

La section 4 présente l'analyse de la stabilité de la coupe en fraisage par intégration de notre modèle de broche dans l'approche de stabilité décrite par BudakAltintas [4]. Un nouveau diagramme de lobes de stabilité est élaboré prenant en compte les propriétés dynamiques réelles des broches aux fréquences de rotation considérées. Une validation expérimentale est réalisée qui montre la pertinence de notre approche.

\section{Description du modèle}

Une analyse technologique a permis d'identifier les éléments structuraux qui contribuent au comportement 


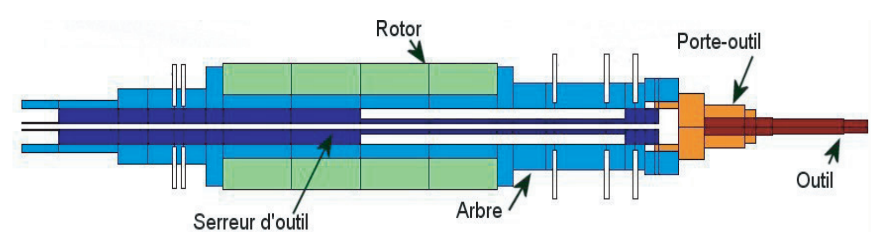

Fig. 2. Modèle éléments-finis de la broche UGV.

dynamique global d'une broche. Ces entités de structure que sont le corps de broche, l'ensemble tournant, le palier arrière ainsi que les liaisons entre elles sont présentées figure 1.

Une caractérisation éléments-finis couplée à une identification modale expérimentale a été conduite sur ces différentes entités [17]. Les résultats montrent une prédominance forte des propriétés dynamiques de l'entité tournante sur le comportement global de la broche. Cette entité est composée de cinq sous-parties qui sont l'arbre, la partie rotorique, le porte-outil, le système de serrage du porte-outil et l'outil (Fig. 2). Le modèle géométrique de l'entité tournante est simplifié de façon à générer un modèle élément-fini de taille acceptable.

Les mouvements de l'entité tournante sont composés de la superposition des mouvements élastiques aux mouvements de corps rigides. Les équations dynamiques sont obtenues par la formulation de Lagrange associée à la méthode éléments-finis. En raison des dimensions des sections de l'arbre les déformations de cisaillement doivent être prises en compte. Les éléments poutres de l'arbre sont dérivés de la théorie des poutres de Timoshenko (G. Genta [18]). Un élément spécifique de poutre-rotor possédant deux nœuds et six degrés de libertés par nœud a été développé. Les équations de Lagrange et les propriétés additives des énergies cinétiques et potentielles permettent d'obtenir les équations dynamiques du rotor $[16]$.

Un rotor en accélération angulaire donne lieu à des matrices comportementales dépendantes du temps (Éq. (1)), mais la résolution du mouvement du rotor en configuration de rotation pseudo-constante peut être décrite à l'aide de matrices linéaires invariantes en fonction du temps (Éq. (2)). Cette modélisation est valide pour la grande majorité des applications. Le modèle élément-fini global de broche est réalisé, de façon classique, par assemblage des matrices élémentaires des éléments constitutifs. Les mouvements du rotor sont combinés à ceux du corps de broche en introduisant les déplacements nodaux relatifs dans les modèles de roulements. Une matrice d'amortissement structural est introduite en utilisant la formulation d'amortissement de Rayleigh. Les équations différentielles du mouvement sont les suivantes :

$$
\mathbf{M}\left(\boldsymbol{q}_{N}\right) \ddot{\boldsymbol{q}}_{N}+\left(\mathbf{C}\left(\boldsymbol{q}_{N} ; \dot{\boldsymbol{q}}_{N}\right)+\mathbf{D}\right) \dot{\boldsymbol{q}}_{N}+K \boldsymbol{q}_{N}=\boldsymbol{F}(t)
$$

dans laquelle $\mathbf{M}$ et $\mathbf{K}$ sont les matrices de masse et de raideur, la matrice $\mathbf{C}$ contient les effets gyroscopiques et centrifuges, D est la matrice d'amortissement structural,

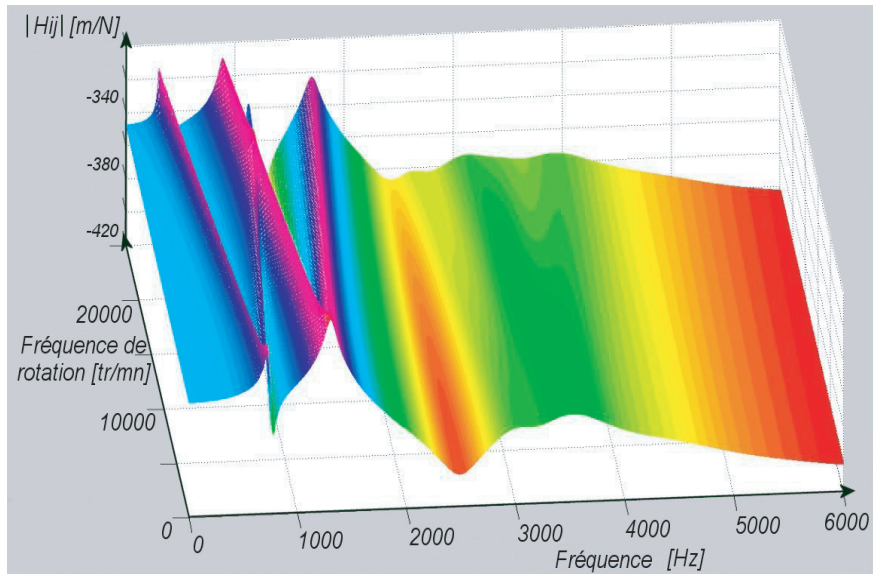

Fig. 3. Graphique 3D de l'amplitude de la réponse en fréquence du nœud en bout d'outil.

$\boldsymbol{q}_{N}$ et $\boldsymbol{F}(t)$ sont les déplacements nodaux et le vecteur des efforts extérieurs, $\boldsymbol{\Omega}$ est la matrice des vitesses angulaires;

$$
\boldsymbol{M}_{0} \ddot{\boldsymbol{q}}_{N}+(2 \boldsymbol{\Omega} \boldsymbol{G}+\mathbf{D}) \dot{\boldsymbol{q}}_{N}+\left(\mathbf{K}-\boldsymbol{\Omega}^{2} \boldsymbol{N}\right) \boldsymbol{q}_{N}=\boldsymbol{F}(t)
$$

dans laquelle $\boldsymbol{M}_{0}$ est la partie constante de la matrice $\mathbf{M}$, $\boldsymbol{G}$ et $\boldsymbol{N}$ proviennent de la décomposition de la matrice $\mathbf{C}$.

Comme la connaissance précise d'une broche n'est en général pas accessible en configuration industrielle, un algorithme de recalage permet le réglage des paramètres matériaux tels que le module d'Young, le module de cisaillement et les coefficients d'amortissement de certaines zones du modèle. Ces paramètres sont réajustés en minimisant l'écart entre la FRF en bout d'outil mesurée expérimentalement et celle du modèle pour une broche à l'arrêt par une procédure d'optimisation et une fonction objectif des moindres carrés [19].

\section{Simulation dynamique du modèle}

Le modèle recalé peut maintenant être simulé pour observer les effets sur le comportement dynamique des grandes fréquences de rotation de la broche. Les FRF du modèle sont déterminées en fonction de la vitesse de broche. Deux boucles numériques de calcul respectivement sur la fréquence d'excitation et sur la vitesse de rotation de la broche permettent l'élaboration d'un graphique 3D (Fig. 3) qui représente la FRF du nœud en bout d'outil en fonction de la vitesse de rotation. Une vue de dessus de ce graphique s'interprète comme un diagramme de Campbell et chaque coupe verticale parallèle au plan $\left(f, H_{i j}(f)\right)$ représente l'évolution de la FRF en fonction de la vitesse.

Les effets dynamiques dus aux forts taux de rotation comme les couplages gyroscopiques et les assouplissements par rotation propre ont une influence significative sur le comportement dynamique de broche. Pour une broche à l'arrêt, deux déformées modales orthogonales sont associées à une même fréquence de vibration 

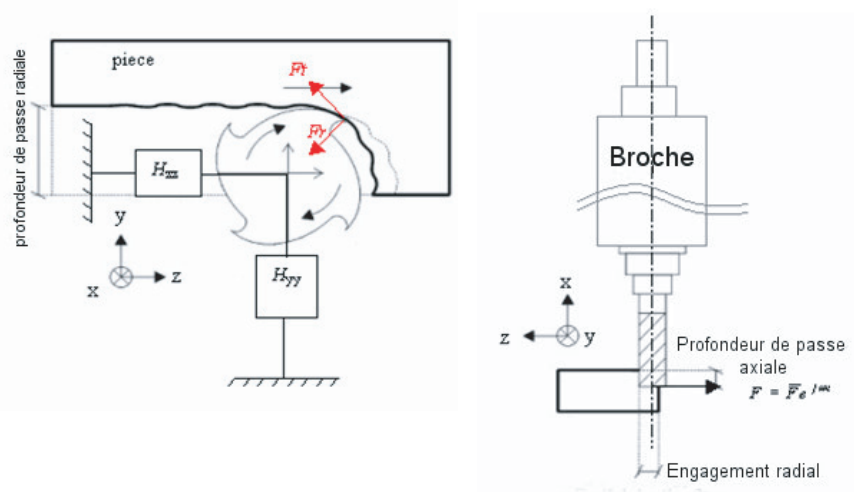

Fig. 4. Vibration auto-entretenue d'une opération de fraisage.

en raison de propriétés d'axisymétrie du rotor. Lorsque la broche tourne, les fréquences associées à ces deux déformées s'écartent l'une de l'autre et de part et d'autre de leur valeur initiale en fonction de la vitesse de rotation du rotor. Ces deux modes sont appelés mode de précession direct et inverse [18]. Une expérience originale [19] permet de valider ces résultats avec une bonne corrélation. Les résultats montrent que les propriétés dynamiques sont fortement dépendantes de la fréquence de rotation. En conséquence, une prédiction dynamique précise nécessite de prendre en compte ces effets de rotation.

\section{Prédiction de la stabilité de la coupe en fraisage}

L'influence du comportement dynamique de la broche sur l'étude de la stabilité d'une opération de fraisage est étudiée. Nous proposons d'intégrer notre modèle dynamique de broche qui est dépendant de sa vitesse de rotation à l'approche de la stabilité en fraisage décrit par Budak \& Altintas [4]. Les relations de stabilité résultant de cette approche sont présentées dans les équations (3) à (5) dans lesquelles $a_{\text {lim }}$ est la profondeur de passe. $Z$ est le nombre de dents de la fraise, $K_{\mathrm{r}}$ le coefficient spécifique de coupe, qui relie la composante radiale de l'effort de coupe $F_{r}$ à la composante axiale de l'effort de coupe $F_{t}$ (Fig. 4). $K_{\mathrm{t}}$ est le deuxième coefficient spécifique de coupe qui relie $F_{\mathrm{t}}$ à l'aire du copeau, $n$ est le fréquence de rotation de la broche, $f_{\mathrm{c}}$ est la fréquence de broutement, et $k$ est un nombre entier correspondant au nombre de lobes à tracer. $H_{i j}(j \omega)$ est la fonction de transfert dépendant de la vitesse de rotation du nœud en bout d'outil. La partie réelle $R_{i j}$ et la phase $\varphi$ de cette fonction sont utiles à la détermination des paramètres de coupe optimum qui optimisent les conditions de débit copeau sans apparition du phénomène de broutement. Un nouveau diagramme de lobe de stabilité est élaboré prenant en compte les propriétés effectives de la broche en rotation.

En pratique la valeur minimum de la partie réelle de la fonction de transfert détermine la valeur de $a_{\text {lim. }}$. La fonction de transfert du nœud représentatif du bout d'outil est déduit de l'équation (2).

$$
\mathbf{H}(j \omega)=\left(-\boldsymbol{M}_{0} \omega^{2}+(2 \boldsymbol{\Omega} \boldsymbol{G}+\mathbf{D}) \omega+\left(\mathbf{K}-\boldsymbol{\Omega}^{2} \boldsymbol{N}\right)\right)^{-1}
$$

$H_{i j}(j \omega)=R_{i j}(\omega)+j I_{i j}(\omega)$ dans laquelle $R_{i j}$ et $I_{i j}$ sont respectivement la partie réelle et la partie imaginaire de la fonction de transfert. $i$ et $j$ représente les numéros des nœuds d'observation et d'excitation.

Les efforts de coupe dans les directions $y$ et $z$ sont,

$$
\{F(t)\}=\left\{\begin{array}{l}
F_{y} \\
F_{z}
\end{array}\right\}=\frac{1}{2} a K_{\mathrm{t}} \boldsymbol{A}_{\mathbf{0}}\{\Delta(t)\} \text { avec } \boldsymbol{A}_{\mathbf{0}}=
$$
$\frac{z}{2 \pi}\left[\begin{array}{ll}\alpha_{y y} & \alpha_{y z} \\ \alpha_{z y} & \alpha_{z z}\end{array}\right]$ la matrice des coefficients directionnels de coupe dépendant de l'immersion de la fraise dans la pièce mais indépendante du temps.

$$
a_{\lim }=\frac{-2 \pi K}{K_{\mathrm{t}} Z} \frac{1}{R(\omega)}
$$

avec $K=f\left(\alpha_{x x}, \alpha_{x z}, \alpha_{z x}, \alpha_{z z}\right)$

$$
n=\frac{60}{T}=\frac{60 \cdot 2 \pi \cdot f_{\mathrm{c}}}{Z(\pi+2 k \pi-2 \varphi)} \text { avec } \varphi=-\tan ^{-1}\left|\frac{I(\omega)}{R(\omega)}\right|
$$

La procédure de détermination des points de stabilité pour chaque vitesse de la broche est la suivante. À chaque fréquence de rotation de la broche est établi un diagramme de lobes de stabilité sur la base des propriétés dynamiques effectives de la broche à cette vitesse. De chacun de ces graphiques intermédiaires n'est extrait que le point dont l'abscisse correspond à la vitesse à laquelle est réalisé le calcul. Il est à noter que si deux points ont la même abscisse (prise en compte de différents modes de la fonction de transfert), on ne garde que celui dont l'ordonnée est la plus petite, puisque l'on cherche les profondeurs de passe maximales admissibles. L'ensemble des points réunis permet d'élaborer un nouveau graphe que nous appellerons le diagramme des lobes dynamiques. L'algorithme de résolution est décrit en référence [19].

L'apport de cette approche est présentée en figure 5. Deux courbes sont présentées qui correspondent à la limite de stabilité basée respectivement sur les propriétés dynamiques d'une broche à l'arrêt et sur les propriétés dynamiques réelles à chaque vitesse de rotation considérée.

Sur la gamme de fréquence de rotation 300012000 tr. min $^{-1}$, on observe des différences significatives de prédiction de stabilité entre les deux approches. La frontière de stabilité, établie par notre approche, fait apparaître de nouvelles zones de stabilité et d'instabilité en comparaison aux lobes construits sur les propriétés statiques de la broche. En particulier, on distingue de nouvelles zones de stabilité autour des fréquences de rotation de 6000,3800 et 3000 tr.min ${ }^{-1}$, et de nouvelles zones d'instabilité autour de 5000 tr. $\mathrm{min}^{-1}$ et au-delà de 7800 tr. $\min ^{-1}$. L'abscisse fréquentielle des pics de stabilité résulte des propriétés modales de l'électrobroche en fonction de sa fréquence de rotation. La hauteur des pics et des creux a varié en fonction de l'évolution de rigidité dynamique de la broche aux vitesses considérées. 


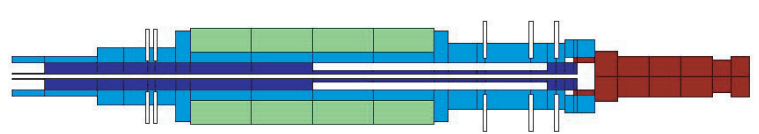

a) Broche d'étude

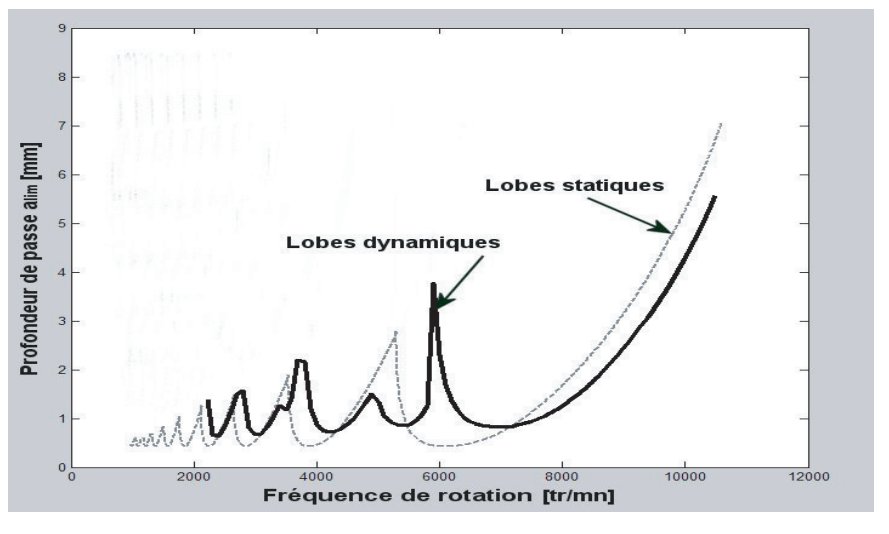

b) Diagramme des lobes de stabilité

Fig. 5. Modèle de broche et diagramme des lobes de stabilité basé sur les propriétés statiques et dynamiques de la broche.

On vérifie sur ce graphique que la prédiction de stabilité par ces deux approches devient similaire lorsque les fréquences de rotation considérées deviennent faibles. En effet, à des taux faibles de rotation le comportement dynamique de la broche tournante tend vers celui de la broche à l'arrêt. La valeur de $a_{\text {lim }}$ tend pour les deux approches vers la valeur minimum prédite par les lobes de stabilité statiques.

\section{Validation expérimentale}

Une démarche expérimentale d'élaboration des lobes de stabilité est mise en place afin d'observer la pertinence de notre modèle. Des rainures sont fraisées à différentes profondeurs axiales et vitesses de broche en prenant une avance à la dent constante de $0,1 \mathrm{~mm} \cdot$ dent $^{-1}$. Cette caractérisation expérimentale est conduite sur un centre d'usinage PCI-SCEMM avec une fraise deux dents de diamètre $16 \mathrm{~mm}$ de marque Jabro JH420 monté dans un porte outil Kelch. La pièce est en aluminium AS7 (Fig. 6). Pour chaque vitesse de broche, une profondeur de passe initiale est choisie de façon à ce qu'il n'y ait pas de broutement. La profondeur est ensuite augmentée jusqu'à l'apparition du phénomène de broutement.

L'identification du phénomène de broutement est réalisée par l'observation et la mesure de plusieurs paramètres. Le premier est le bruit généré par la coupe qui est mesuré par un microphone. Le signal enregistré est ensuite traité par l'intermédiaire du logiciel Accordmill [20]. La fréquence de rotation de la fraise $f_{\mathrm{r}}$ et ses harmoniques ainsi que la fréquence de passage des dents $f_{\mathrm{t}}$ et ses harmoniques sont soustraites du signal. Le bruit environnant est aussi filtré par soustraction du son avant usinage. Les pics fréquentiels restants sont identifiés comme des fréquences de broutement (Fig. 7). Ces mesures sont

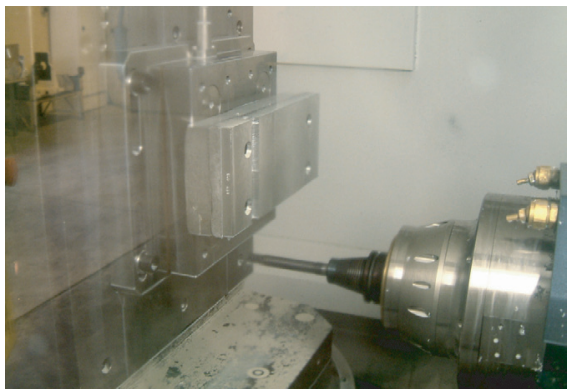

a) Configuration d'usinage

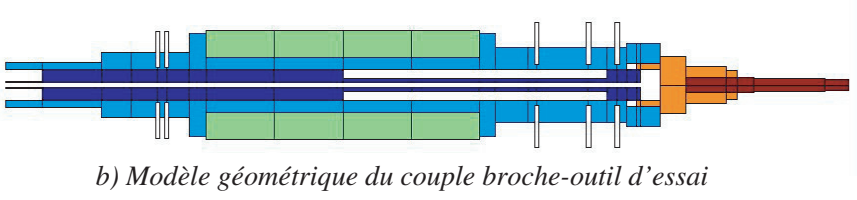

Fig. 6. Configuration d'essai et modèle associé de broche.

combinées avec une interprétation visuelle de l'état de surface de la rainure usinée. Les résultats issus des essais expérimentaux sont ensuite comparés avec les lobes calculés numériquement. La figure 7 présente sur un même graphique les points expérimentaux mesurés ainsi que les diagrammes des lobes de stabilité statiques et dynamiques.

Les résultats des mesures expérimentales sont comparés avec les lobes de stabilité obtenus par le modèle. Le premier pic est prédit de façon précise (jusqu'à 10000 tr. min $^{-1}$ ) puis on observe un décalage vers la droite des pics du modèle par rapport aux mesures. La position de la courbe limite de stabilité prédite par le modèle semble globalement plus précise que celle obtenue par une approche construite sur les propriétés de la broche à l'arrêt. Une source d'explication du décalage observé entre les lobes peut être trouvée dans les écarts résiduels restant à l'issue de la procédure de recalage modal et harmonique du modèle élément-fini. En effet une erreur de prédiction modale sur le modèle se traduit par une évolution de la fonction de transfert de la broche et induit une variation sur la stabilité de la coupe en usinage.

\section{Conclusion}

Dans cet article, un modèle élément-fini d'une broche en rotation est développé afin de caractériser le comportement dynamique d'une broche UGV. Les résultats d'analyse montrent une dépendance du comportement dynamique de la broche avec sa vitesse de rotation. Les FRF de la broche tournante sont ensuite utilisées pour construire analytiquement la limite de stabilité en fraisage.

La position des lobes de stabilité construits à partir du modèle sont plus proches des mesures expérimentales que ceux construits sur les propriétés dynamiques de la broche à l'arrêt. On montre en particulier que l'ancienne approche conduit à une sous-prédiction de la profondeur 


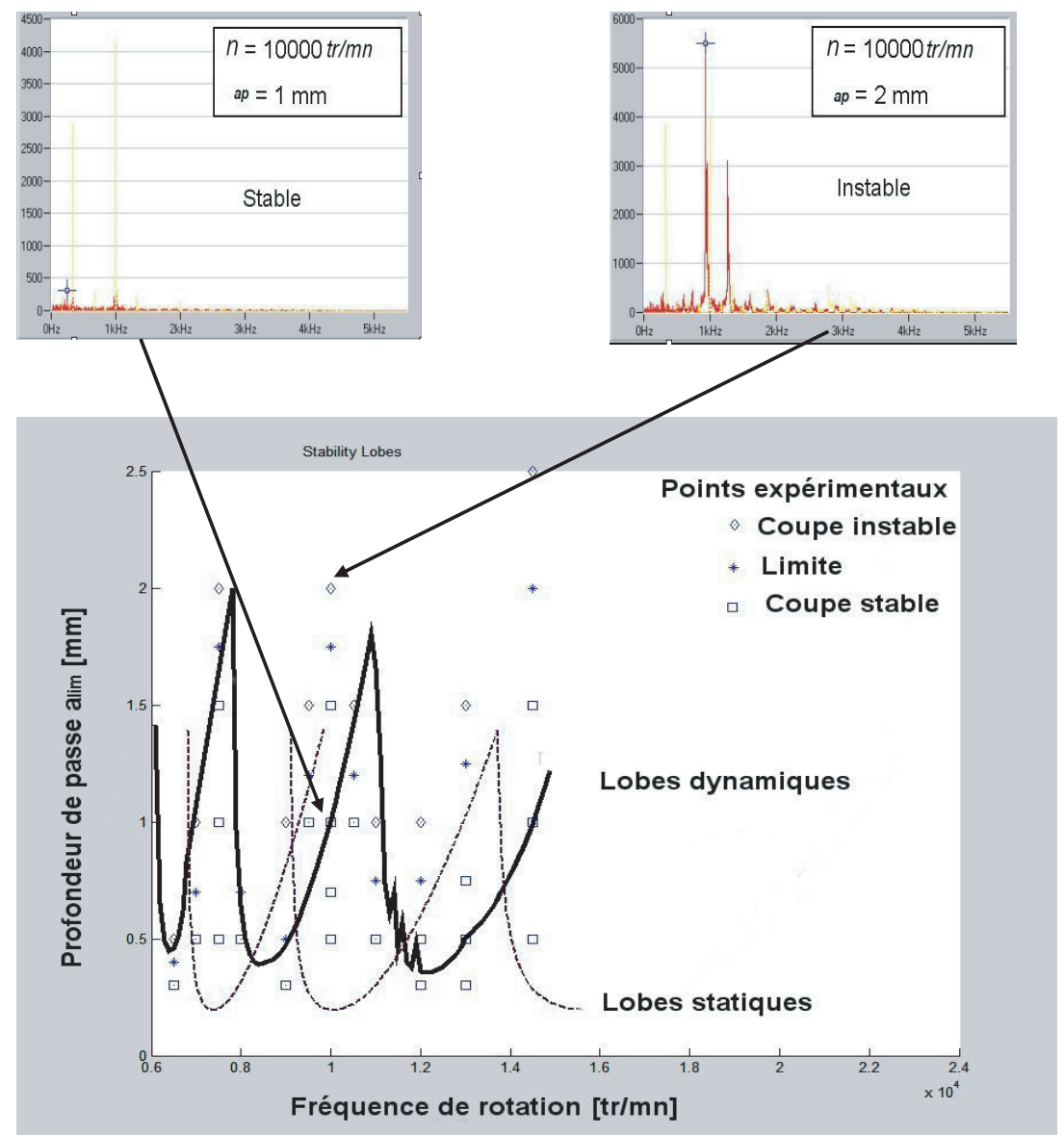

Fig. 7. Lobes de stabilité «statiques », lobes de stabilité «dynamiques » et points de mesure expérimentaux.

de coupe possible sans broutement et conduit dans certains cas à une mauvaise prédiction de la frontière de stabilité. Une amélioration réelle est apportée par la prise en compte des propriétés dynamiques de la broche à sa vitesse effective, dans la prédiction de la limite de stabilité en fraisage. Cependant des améliorations restent à apporter à la procédure de recalage modal et harmonique du modèle élément-fini pour affiner encore la prédiction.

Enfin, cette procédure a été développée pour une utilisation industrielle. Le modèle de connaissance sur lequel elle est construite permet la modélisation de tout type de broche. Les conditions d'usinage du couple broche-outil peuvent être optimisées afin de garantir d'une part la tenue en service de l'équipement et d'autre part les conditions optimales de coupe en terme de débit copeaux et qualité de la surface.

Remerciements. Ces travaux de recherche s'inscrivent dans le cadre de la Fédération de Recherche TIMS/CNRS 2856 financée par le Conseil Régional d'Auvergne, le Ministère de la Recherche, le CNRS et le CEMAGREF.

\section{Références}

[1] S. Tobias, W. Fishwick, Theory of regenerative machine tool chatter, The Engineer, February 1958
[2] J. Tlusty, M. Polacek, The stability of machine tools against self-excited vibrations in machining, Proceedings of the ASME International Research in Production Engineering, Pittsburgh, USA, 1963, pp. 465-474

[3] H. Merritt, Theory of self-excited machine tool chatter, Transactions of ASME J. Eng. Industry 87 (1965) 447454

[4] Y. Altintas, E. Budak, Analytical prediction of stability lobes in milling, Annals of the CIRP 44/1 (1995) 357-362

[5] J. Agapiou, E. Rivin, C. Xie, Toolholder/Spindle Interfaces for CNC Machine Tools, Annals of the CIRP 44/1 (1995) 383-387

[6] S. Smith, P. Jacobs, J. Halley, The effects of drawbar force on metal removal rate in milling, Annals of the CIRP 48/1 (1999) 293-296

[7] W.R. Wang, C.N. Chang, Dynamic analysis and design of a machine tool spindle-bearing system, J. Vibration Acoustics 116 (1994) 280-285

[8] M.A. Alfares, A. Elsharkawy, Effects of axial preloading of angular contact ball bearings on the dynamics of a grinding machine spindle system, J. Mat. Proc. Tech. 136 (2003) 48-59

[9] T.C. Lim, Vibration transmission through rolling element bearing, Part I to IV, J. Sound Vibration 139 (1990) 179199

[10] B.R. Jorgensen, Y.C. Shin, Dynamics of spindle-bearing systems at high speeds including cutting load effects, J. Manufacturing Sci. Eng. 120 (1998) 387-394 
[11] J.L. Stein, J.F. Tu, Active thermal preload regulation for machine tool spindles with rolling element, J. Manufacturing Sci. Eng. 118 (1996) 499-505

[12] B. Bossmanns, J.F. Tu, A thermal model for high speed motorized spindles, Int. J. Machine Tools Manufacture 39 (1999) 1345-1366

[13] S.A. Spiewak, T. Nickel, Vibration based preload estimation in machine tool spindles, Int. J. Machine Tools Manufacture 41 (2001) 567-588

[14] R.P.H. Faassen, N. van de Wouw, J.A.J. Oosterling, H. Nijmeijer, Prediction of regenerative chatter by modelling and analysis of high-speed milling, Int. J. Machine Tools Manufacture 43 (2003) 1437-1446

[15] T. Schmitz, J. Ziegert, C. Stanislaus, A method for predicting chatter stability for systems with speeddependent spindle dynamics, Society of manufacturing engineers technical paper TPO4PUB182, Transactions of NAMRI/SME 32 (2004) 17-24
[16] V. Gagnol, C.B. Bouzgarrou, P. Ray, C. Barra, Dynamic analysis of a high speed machine tool spindlebearing system, CD-ROM proceedings of the ECCOMAS Conferences, Madrid, Spain, June 21-24 (2005)

[17] V. Gagnol, C.B. Bouzgarrou, P. Ray, C. Barra, Modelling approach for a high speed machine tool spindle-bearing system, CD-ROM Proceedings of the International Design Engineering Technical Conferences, Long Beach, USA, September 24-28 2005

[18] G. Genta, Vibration of structures and Machines, Practical Aspects, Springler-Verlag, 1995

[19] V. Gagnol, B.C. Bouzgarrou, P. Ray, C. Barra, Modelbased chatter stability prediction for high-speed spindles, Int. J. Machine Tools Manufacture 47 (2007) 1176-1186

[20] Accord-mill software from ELPS company, 25 rue Coulomb, 46100 Figeac 